\title{
Apprendre Autrement l'Electrotechnique en Utilisant l'Anglais Technique dans le Cadre du Nouveau PPN des IUT GEII
}

\author{
M.E.H. Benbouzid \\ Département de GEII, IUT de Brest, Université de Bretagne Occidentale \\ Rue de Kergoat, CS 93837, 29238 Brest Cedex 03 \\ m.benbouzid@ieee.org
}

\begin{abstract}
Résumé : Il s'agit dans cet article de décrire une expérience menée au sein du Département Génie Electrique et Informatique Industrielle de l'IUT de Brest, dans le cadre de la mise en œuvre du nouveau programme pédagogique national de 2005. L'expérience menée concerne plus particulièrement les nouveaux Modules Complémentaires dits Apprendre Autrement. Nous avons donc mis à profit ce type de module pour essayer de redorer le blason de l'électrotechnique qui connaît depuis un certain nombre d'années une désaffection continue bien que les besoins ne fassent que s'accroître au niveau industriel (métiers de l'électrotechnique). Dans ce contexte difficile, un investissement important est nécessaire pour rendre l'attractivité que mérite l'électrotechnique; au travers d'un gros effort d'amélioration de son image. L'expérience menée à Brest, dans le cadre de la première année en IUT GEII, consiste à utiliser l'anglais technique comme source de motivation pour intéresser les étudiants à l'électrotechnique. Nous avons, par ailleurs, mis à profit ce module pour mettre en œuvre une expérience menée au Royal Institute of Technology à Stockholm (Suède) et qui consistait à expliquer les fondements de l'électrotechnique à des novices au travers de l'apprentissage par analogie avec des systèmes de la vie courante.
\end{abstract}

Mots-clés : Nouveau programme pédagogique national (ppn) des IUT, module apprendre autrement, électrotechnique, anglais technique.

\section{INTRODUCTION}

Dans le cadre du nouveau Programme Pédagogique National (PPN 2005) de la spécialité Génie Electrique et Informatique Industrielle (GEII) [1], il a été mis en place des Modules Complémentaires dits Apprendre Autrement qui avaient pour objectifs d'aborder autrement une même matière dans laquelle l'étudiant a plus ou moins échoué.

Constatant le déclin continu de l'attractivité de l'électrotechnique, soit pour des raisons d'image soit pour des raisons de difficultés de maitrise des concepts de base ; le département GEII de l'IUT de Brest a décidé de mettre à profit ce type de module pour essayer de redorer le blason de l'électrotechnique au travers d'un gros effort d'amélioration de son image. L'expérience menée à l'IUT de Brest, dans le cadre de la première année, consiste à utiliser l'anglais technique comme source de motivation pour intéresser les étudiants à l'électrotechnique. Il est évident que l'objectif d'un tel module devient double, puisqu'il permet aussi d'introduire l'étudiant à l'anglais technique et professionnel.

Nous avons, par ailleurs, mis à profit ce module pour mettre en œuvre une expérience menée au Royal Institute of Technology à Stockholm (Suède) et qui consistait à explique les fondements de l'électrotechnique à des novices au travers d'applications particulières [2-3].

\section{CONTENU DU MODULE}

Le module tel que proposé aux étudiants a été construit sur la base d'une suite logique de chapitres.

\subsection{Chapitre I : En Guise d'Introduction}

Dans cette première partie, il est présenté aux étudiants un certain nombre de formes grammaticales et lexicales qui existent dans la langue usuelle, mais dont l'accumulation donne au texte technique une forme qui n'est pas toujours dépourvue de difficultés. Plus particulièrement, nous avons abordés les thèmes suivants :

- Noms composés ;

- Adjectifs composés ;

- Les formes elliptiques.

Pour illustration, un exemple d'exercice que les étudiants ont à traiter : Former des noms composés avec les expressions anglaises suivantes.

a) An engine with six pistons;

b) A reference which has been mentioned above;

c) The range at which it can operate;

d) A tube used as rectifier and filled with gas;

f) Materials which are mechanically and thermally resistant;

g) Investments that enable to reduce costs. 


\subsection{Chapitre II : Electrical Safety}

Ce chapitre est exploité pour introduire une grande partie de la nomenclature anglaise en électrotechnique. En effet, on y présente les notions de base de l'électricité, des risques électriques et les moyens de protections.

Ce chapitre et ceux qui suivent sont systématiques organisés en deux parties :

- Understanding the text ;

- Language study.

Pour illustration, un exemple d'exercice que les étudiants ont à traiter: According to figure 1, fill in the blanks with words from list 1, or list 2, or list 3.

List 1: unless, is composed of, however, consequently, by means of, so that, as a result.

List 2: coils, wound, trigger, control, toroid, electromagnet, push button.

List 3: toroid, short-circuit, leakage current, emf, arcing, trigger.

This circuit breaker ...... a magnetic circuit in the shape of a ..... with two ......, which are ..... around it. A third coil is linked to an ......, which itself can ...... off the circuit. There are also two ...... switches to ...... the circuit manually.

..... there is any ......, the flow in both main coils is equal and ...... nothing happens. ...... if there is a ...... somewhere, the flow is unbalanced, and an electromagnetic field appears in the ......, thus creating an ...... in the test coil ...... the electromagnet is actuated which, ...... a mechanical ......, cuts the current off, while ensuring rapid separation of the contacts ...... no dangerous ...... may happen.

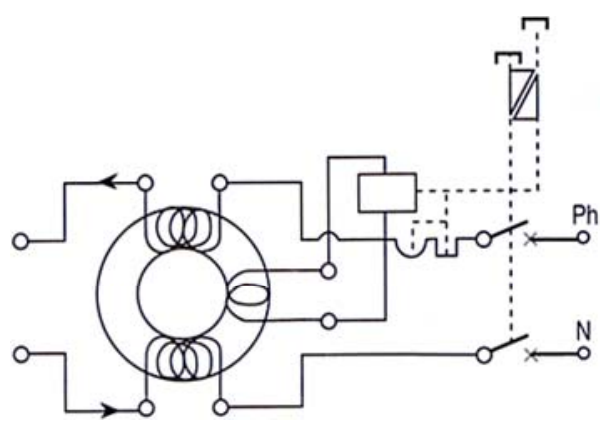

Fig. 1. A circuit breaker.

\subsection{Chapitre III : Conductors, Resistance, and Semi-Conductors}

Ce chapitre est la suite logique du chapitre II. En effet, on y aborde les notions de résistance, de conducteur, et de semi-conducteurs (brièvement).

Il faut noter que, progressivement, les étudiants sont sensibilisés à la prononciation principalement au travers de la lecture d'équations liés à l'électrotechnique. Pour illustration, un exemple d'exercice que les étudiants ont à traiter :

1. Give the expression of the following equations.

a) $\sigma$ equals root of $\mathbf{1}$ over $\boldsymbol{N}$ by the summation from 1 to $\boldsymbol{N}$ of $\boldsymbol{x}$ sub $\boldsymbol{i}$ minus $\boldsymbol{m}$ all to the square. b) a squared over $\boldsymbol{b}$ cubed equals $\boldsymbol{k}$.

2. Write how you should read the following equations.

a) $E_{c}=\frac{1}{2} M V^{2}$

b) $T=2 \pi \sqrt{\frac{l}{g}}$

\subsection{Chapitre IV : Rotating Machines}

Ce chapitre est mis à profit pour expliquer aux étudiants le principe de fonctionnement des machines à courant continu et les éléments de base du redressement. Ceci s'est principalement fait par l'intermédiaire d'exemples et d'applications ludiques tels que celui illustré par la figure 2 [4].

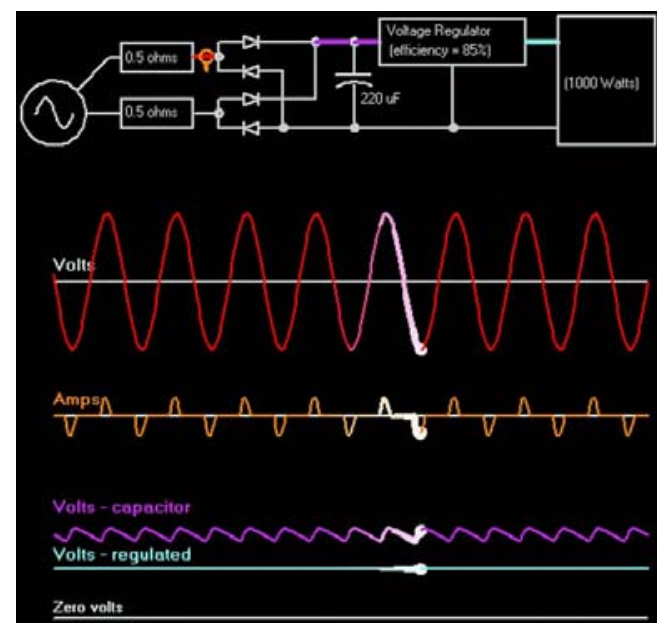

(a) A modern single-phase rectifier-capacitor supply.

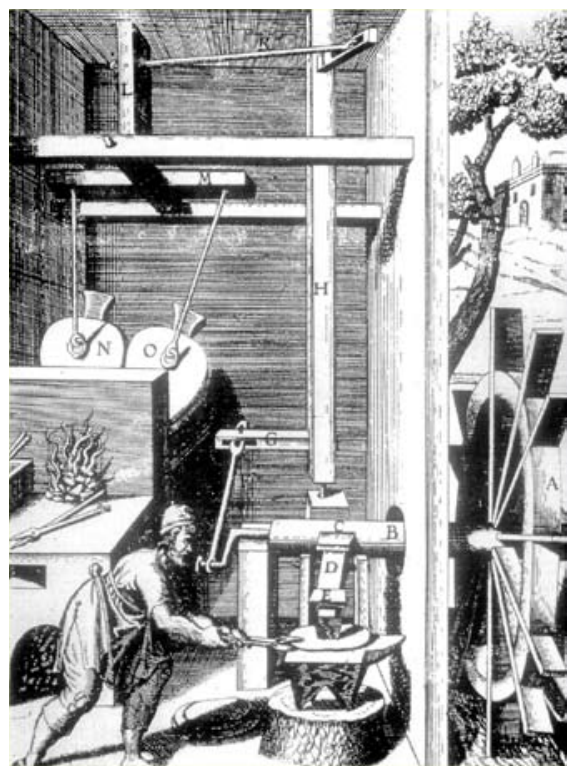

(b) An early single-pulse water-wheel load. The trip toggle $C$ on the shaft B lifts, and suddenly drops, the hammer E. But, like a single-phase rectifier, the hammer only extract energy during a short part of each cycle.

Fig. 2. [4]. 
Pour illustration, un exemple d'exercice que les étudiants ont à traiter: Describe the circuit of figure 3 using the following phrases: centre-tapped transformer, secondary winding, load resistor, current is applied to ..., forward/reverse bias.

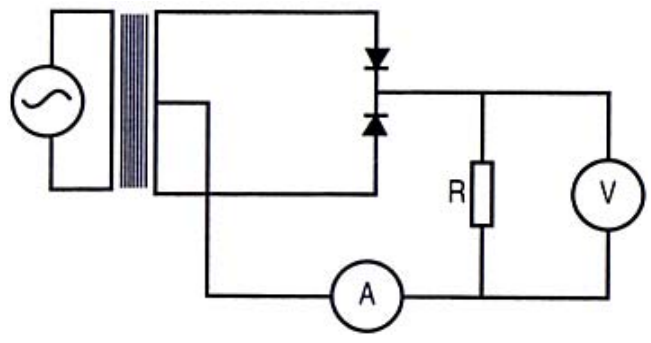

Fig. 3.

\subsection{Chapitre V : $\quad$ Explaining Power System Operation to Nonengineers}

Ce dernier chapitre est la mise en œuvre pratique à Brest d'une expérience menée au Royal Institute of Technology à Stockholm (Suède) et qui consistait à explique les fondements de l'électrotechnique à des novices au travers d'une application bien particulière [2].

Il s'agit dans notre cas d'utiliser un vélo (tandem particulier) tel qu'illustré par la figure 4, pour permettre aux étudiants de bien visualiser et de mieux comprendre les phénomènes de base d'un système électrique (réseau électrique).

\subsubsection{Représentation de base d'un réseau électrique}

On considère le système tandem roulant à vitesse constante de la figure 5 . Le but de ce système est de maintenir les personnes en bleu à l'arrière du vélo en mouvement, même s'ils ne participent pas à la génération de la puissance nécessaire au mouvement du vélo : Ils représentent alors la charge. Les personnes qui pédalent (en rouge) permettent de générer la puissance nécessaire au mouvement du vélo: Ils représentent les centrales électriques. La chaîne connectant tout les éléments du vélo est équivalente au réseau électrique (lignes HT pour le transport de la puissance électrique). Cette chaîne doit impérativement tourner à vitesse constante ce qui est l'analogue d'un réseau électrique ayant une fréquence constante. Plus particulièrement, au niveau des analogies, la chaîne supérieure sous tension constante est équivalente à une ligne de transport à tension constante.
La chaîne inférieure, quant à elle, n'est pas sous tension, ce qui nous permet de dire qu'elle est équivalente au neutre. Pour terminer, l'élément de transmission est l'équivalent d'un transformateur reliant les centrales de génération au réseau électrique.

Cette représentation est alors utilisée pour expliquer aux étudiants un certains nombre de notions. Il est bien évident, qu'avec celle-ci, il n'est pas possible de trouver une analogie avec n'importe quelle notion d'électrotechnique. Dans ce qui suit, nous y présentons les principales.

\subsubsection{Différents types de centrales électriques}

Si un personnage rouge est connecté à la chaîne par une seule transmission (figure 6a), il doit constamment pédaler à la même vitesse et avec la même puissance pour maintenir une vitesse constante: Ceci est bien évidement l'analogue d'une centrale de base traditionnelle.

Dans le cas d'un système à plusieurs rapports (figure $6 \mathrm{~b})$, le personnage rouge peut pédaler moins vite : Ceci est maintenant l'analogue d'une centrale intermédiaire et plus particulièrement une centrale hydraulique dont la génération dépend du flux de la rivière.

Enfin, le personnage rouge peut ne pédaler que lorsqu'il fait beau par exemple. Ceci pourrait être l'analogue d'une éolienne qui ne fonctionnerait que lorsque la vitesse du vent n'est ni très faible ni très élevée. Cette éolienne complète bien sur la génération des autres centrales (figure 6c).

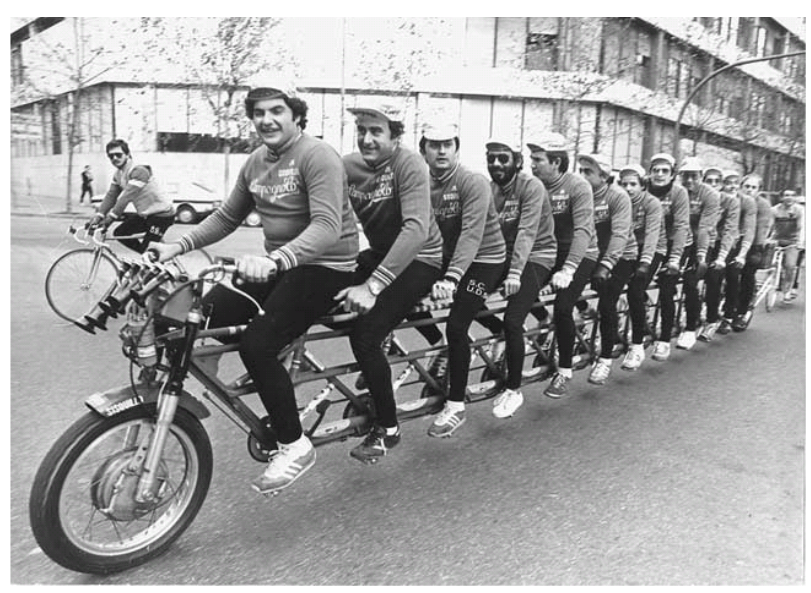

Fig. 4. [2].

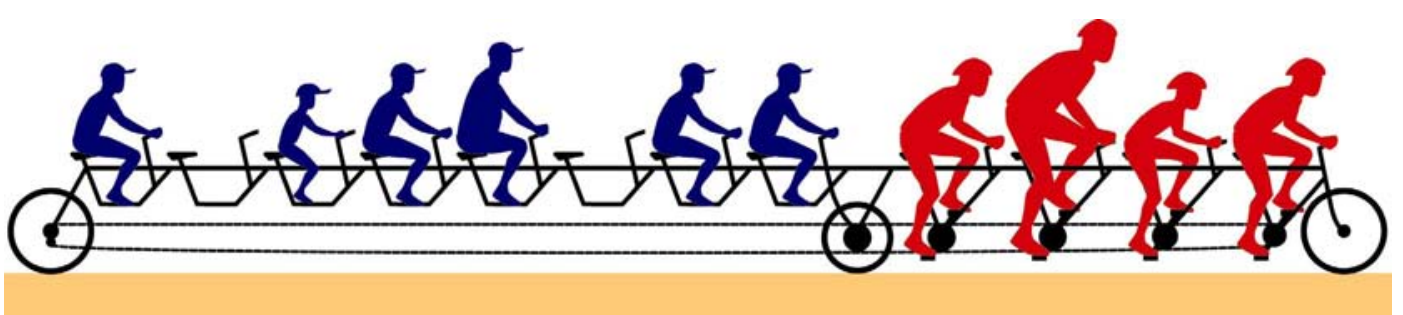

Fig. 5. Représentation de base d'un réseau électrique. 


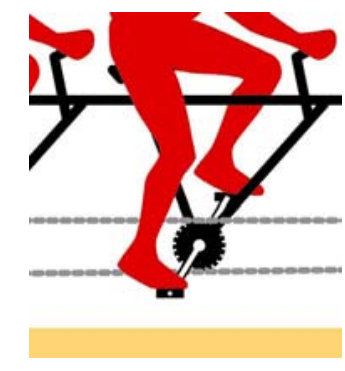

(a) Centrale de base traditionnelle (ex. : Centrale nucléaire).

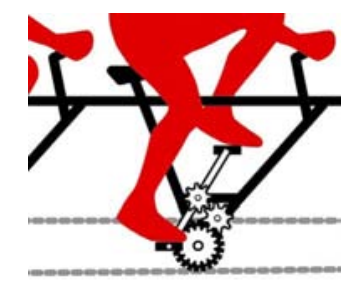

(b) Centrale intermédiaire (ex. : Centrale hydraulique).

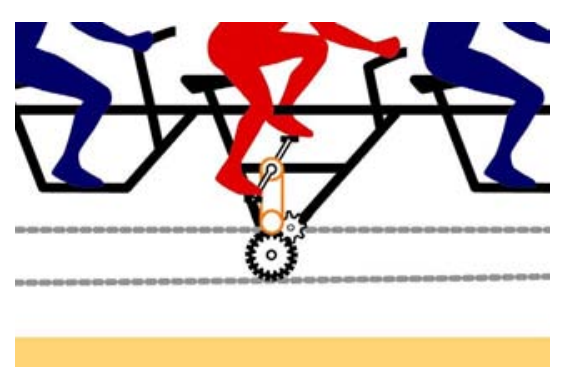

(c) Eolienne.

Fig. 6. Analogies avec les centrales de génération d'électricité.

\subsubsection{Différents types de charges}

L'analogie avec la notion de charge électrique dans un circuit et plus généralement dans un réseau électrique est mise en évidence par la figure 7. En effet, la figure 7a, montrant une phase de freinage, illustre des résistances électriques (ex. : éclairage et la majorité des systèmes de chauffage électrique). La figure $7 \mathrm{~b}$, montrant une personne debout et bloquant les pédales tout en étant entraînée, illustre des charges actives du type moteur électrique. Enfin, la figure 7c, montrant une personne inclinée, illustre une charge inductive. Il sera bien sûr nécessaire de compenser l'effet de ce type de charge (ex. : moteur électrique, bobines d'induction, etc.).

\subsubsection{La puissance réactive et sa compensation}

Comme l'illustre la figure $7 \mathrm{c}$, une charge inductive introduit un déphasage. Plus particulièrement, elle introduit un retard qu'il faudra compenser.

La notion de compensation est illustrée par la figure 8 . En effet, le personnage rouge (l'analogue d'une centrale électrique) doit s'incliner de l'autre côté pour compenser l'inclinaison du personnage bleu (l'analogue d'une charge inductive). Ceci illustre parfaitement le principe de la compensation en utilisant un compensateur synchrone.

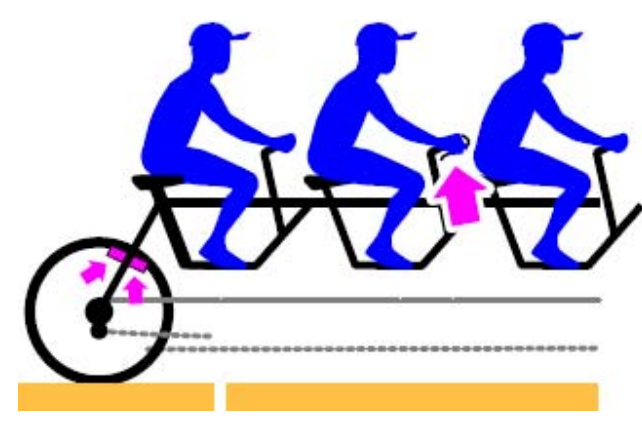

(a) Charges du type résistance électrique.

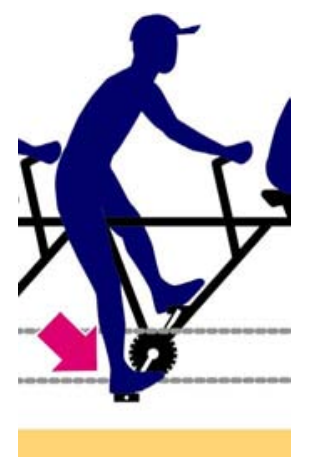

(b) Charges actives du type moteur électrique.

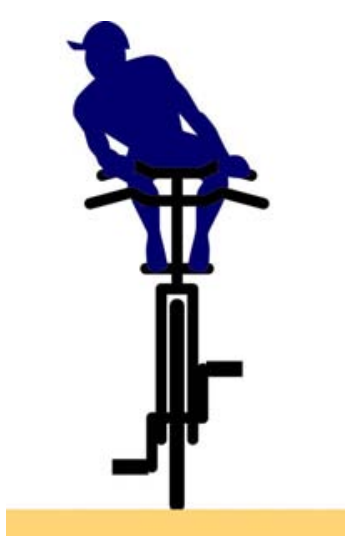

(c) Charges inductives.

Fig. 7. Analogies avec différents types de charges.

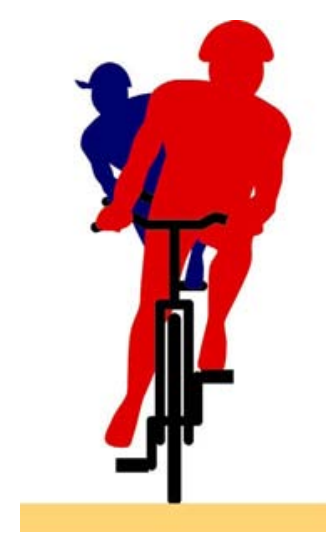

Fig. 8. Principe de la compensation de la puissance réactive. 


\section{CONCLUSIONS}

L'expérience menée a bien atteint ses objectifs puisque le retour des étudiants est très positif. En effet, l'apprentissage par analogie avec des systèmes de la vie courante a permit aux étudiants de bien visualiser et de mieux appréhender certaines notions de base de l'électrotechnique, entre autres des notions abstraites telles que la puissance réactive et sa compensation. D'un autre côté, ce module leur a permit aussi de commencer à ce familiariser avec l'anglais technique et professionnel.

Cette expérience n'a malheureusement été menée que pour un groupe de 12 étudiants. En effet, d'un point de vue organisation, il était impossible de l'envisager pour toute la promotion. Cependant, le principe a été étendu cette année à la nouvelle Licence Professionnelle de l'IUT GEII pour l'option Electrotechnique et Electronique de Puissance. Il est bien évident, que dans ce cadre sont abordés des notions plus avancées de l'électrotechnique. Pour illustration, la figure 9 illustre comme a été abordée la notion de distorsion harmonique dans un réseau électrique.

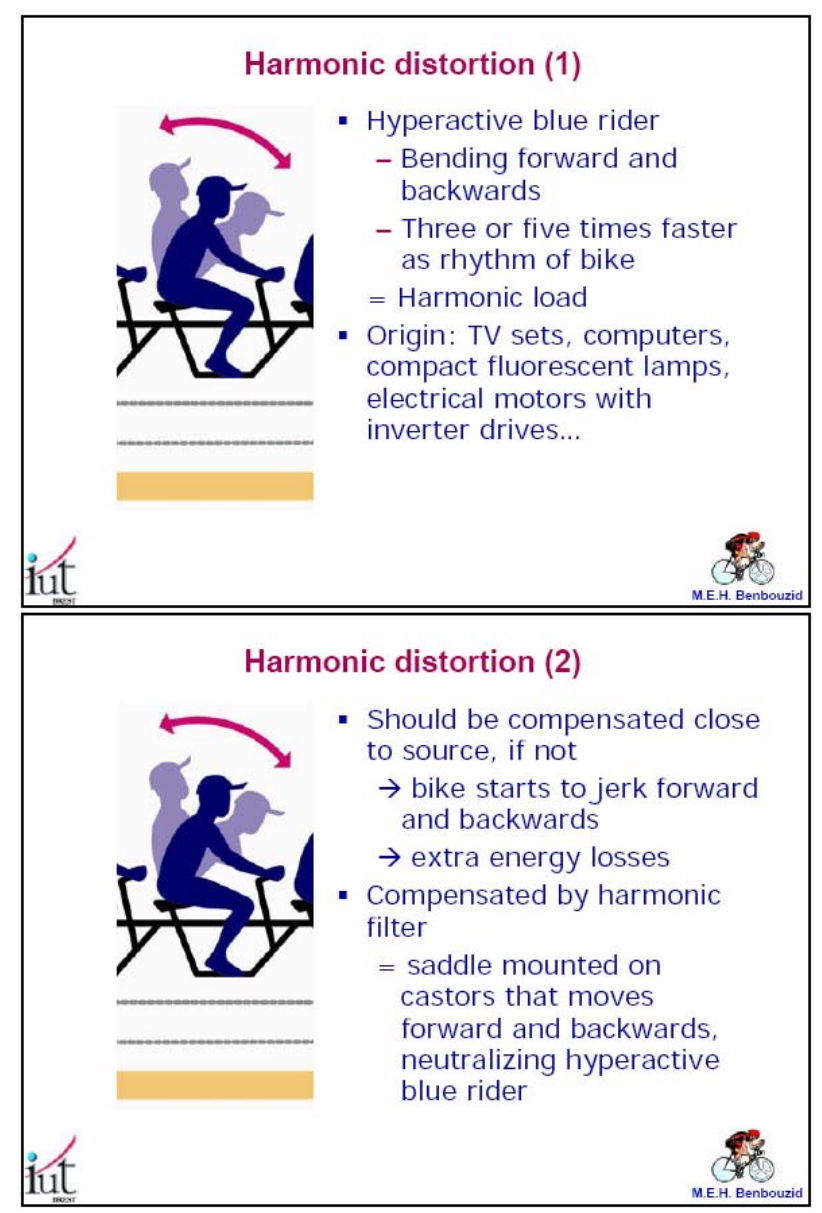

Fig. 9. Vulgarisation de la notion de distorsion harmonique.

\section{Remerciements}

Je tiens tout particulièrement à remercier Jean-Luc Mourrain, Chef du Département GEII de l'IUT de Brest, d'avoir soutenu la mise en place de ce module et aussi d'avoir permis sa pérennisation en Licence Professionnelle.

\section{Références Bibliographiques}

[1] http://media.education.gouv.fr/file/76/8/768.pdf.

[2] L. Söeder, "Explaining power system operation to non-engineers," IEEE Power Engineering Review, vol. 22, N4, pp. 25-27, April 2002.

[3] S. Fasbinder and B. de Wachter, "The electrical system as a tandem bicycle," Leonardo Energy, www.leonardo-energy.org, September 2006.

[4] A. McEachern, "Electrical diversions and amusements. Six pulses rectifiers, five century ago," Leonardo Energy, www.leonardo-energy.org, May 2006. 